\title{
Uso do Coping Religioso/Espiritual diante das Toxicidades da Quimioterapia no Paciente Oncológico
}

\author{
Cancer Patient use of Religious/Spiritual Coping to Deal with the Toxicities of Chemotherapy \\ Uso del Coping Religioso/Espiritual ante las Toxicidades de la Quimioterapia en el Paciente Oncológico
}

Leomar Santos Moraes Filho'; Hilma Tereza Tôrres Khoury²

\begin{abstract}
Resumo
Introduçáo: $\mathrm{O}$ adoecimento por câncer é considerado um grave problema de saúde pública e um evento estressante na vida dos pacientes. Isso porque é significativo o impacto emocional diante do diagnóstico e o desgaste psíquico durante o tratamento, sobretudo no que toca à quimioterapia antineoplásica, que expôe o paciente a toxicidades indesejáveis. Nesse sentido, emerge a necessidade de mobilização de recursos pessoais adaptativos para lidar com os diversos desconfortos, processo este compreendido como coping, podendo ainda estar associado ao uso da religião, espiritualidade ou fé (coping religioso/espiritual). Objetivo: Investigar a relação entre o uso do coping religioso/ espiritual e as respostas orgânicas às toxicidades da quimioterapia. Método: Estudo analítico, observacional, com corte transversal e delineamento correlacional. A amostra náo probabilística foi composta por 40 pacientes da Unidade de Alta Complexidade em Oncologia de um Hospital Universitário. Como instrumentos, foram utilizados um questionário sociodemográfico e de saúde, e a escala de coping religioso/espiritual. Resultados: De um modo geral, o coping religioso/espiritual tanto positivo como negativo foi moderadamente utilizado por todos os participantes, independentemente do nível de toxicidades. Conclusáo: A partir dos resultados encontrados, conclui-se que os pacientes com câncer submetidos à quimioterapia fazem uso tanto de estratégias de coping positivas como negativas, sendo as positivas utilizadas em maior intensidade.
\end{abstract}

Palavras-chave: Tratamento Farmacológico; Espiritualidade; Toxicidade.

\begin{abstract}
Introduction: Cancer is considered a serious public health problem and a stressful event in the lives of patients. That is because the emotional impact of the diagnosis and the psychological damage during treatment are significant, especially in patients undergoing antineoplastic chemotherapy, which exposes them to undesirable toxicities. Therefore, cancer patients need to marshal adaptive personal resources to deal with various types of discomfort, a coping process that can be associated with a reliance on religion, spirituality, or faith (religious/spiritual coping). Objective: To investigate the relationship between the use of religious/spiritual coping and the organic responses to the toxicities of chemotherapy. Method: This was an analytical, observational, cross-sectional, correlational study. Using non-probability sampling, we selected 40 patients under treatment in the high complexity oncology unit of a university hospital. We applied a custom sociodemographic/health questionnaire and the brief religious coping scale. Results: In general, religious/spiritual coping, positive and negative, was moderately employed by all participants, regardless of the degree of the toxicities. Conclusion: We conclude that cancer patients undergoing chemotherapy use positive and negative coping strategies, although positive coping was used to a greater degree in our sample.

Key words: Drug Therapy; Spirituality; Toxicity.
\end{abstract}

Resumen

Introducción: La enfermedad por cáncer se considera un grave problema de salud pública y un evento estresante en la vida de los pacientes. Esto es porque es significativo el impacto emocional ante el diagnóstico y el desgaste psíquico durante el tratamiento, sobre todo, en lo que toca a la quimioterapia antineoplásica, que expone al paciente a toxicidades indeseables. En este sentido, emerge la necesidad de movilización de recursos personales adaptativos para lidiar con las diversas incomodidades, proceso este comprendido como coping, pudiendo aún estar asociado al uso de la religión, espiritualidad o fe (coping religioso/espiritual). Objetivo: Investigar la relación entre el uso del coping religioso/espiritual y las respuestas orgánicas a las toxicidades de la quimioterapia. Método: Estudio analítico, observacional, con corte transversal y delineamiento correlacional. La muestra no probabilística fue compuesta por 40 pacientes de la Unidad de Alta Complejidad en Oncología de un Hospital Universitario. Como instrumentos se utilizaron un cuestionario socio demográfico y de salud y la escala de coping religioso-espiritual. Resultados: En general, el coping religioso espiritual, tanto positivo como negativo, fue moderadamente utilizado por todos los participantes, independientemente del nivel de toxicidades. Conclusión: A partir de los resultados encontrados se concluye que los pacientes con cáncer sometidos a quimioterapia hacen uso tanto de estrategias de coping positivas como negativas, siendo las positivas utilizadas en mayor intensidad. Palabras clave: Tratamiento Farmacológico; Espiritualidad; Toxicidad.

${ }_{1}^{1}$ Psicólogo-Residente. Programa de Residência Multiprofissional em Saúde (PRMS)/Hospital Universitário João de Barros Barreto (HUJBB)/ Universidade Federal do Pará (UFPA). Belém (PA), Brasil. E-mail: leomoraesfh@gmail.com.

${ }_{2}^{2}$ Psicóloga. Professora-Associada e Pesquisadora. Faculdade de Psicologia e PRMS/UFPA. Belém (PA), Brasil. E-mail: hilmatk@yahoo.com.br.

Endereço para correspondência: Leomar Santos Moraes Filho. Unidade 203, Rua 6, nº 5 - Cidade Operária. São Luís (MA), Brasil. CEP 66073-160. 


\section{INTRODUÇÃO}

Câncer é o nome dado a um conjunto de mais de cem doenças em que células anormais do corpo multiplicam-se e espalham-se de maneira descontrolada com possibilidade de invasáo dos órgãos e tecidos, podendo ainda se disseminarem para outras regiôes do corpo por meio de processos metastáticos ${ }^{1,2}$. Apesar do caráter milenar da doença, é com o processo de industrialização que o padrão de saúde e de patologias da população é modificado, gerando o aumento da incidência de câncer ${ }^{3}$ e tornando-o um grave problema de saúde pública mundial.

O adoecimento por câncer é também um evento potencialmente estressor. Isso porque é considerável o impacto emocional diante do diagnóstico e o desgaste psíquico durante o tratamento, sobretudo em relação à quimioterapia antineoplásica que expóe o paciente a diversas toxicidades. Por toxicidades, entende-se a capacidade inerente e potencial do agente tóxico de provocar efeitos nocivos em organismos vivos ${ }^{4}$. Os agentes quimioterápicos não possuem especificidades; agridem simultaneamente células cancerosas e células normais de rápida proliferação, incidindo particularmente sobre o tecido hematopoiético (medula óssea), germinativo, folículo piloso e aparelho gastrintestinal, por apresentarem elevada atividade mitótica ${ }^{5}$. Assim sendo, a experiência da quimioterapia impóe que o paciente, na maioria dos casos, conviva com os efeitos tóxicos indesejáveis, tais como: queda de cabelo (alopecia), náuseas, vômitos, perda ou diminuição da força física (astenia), diarreia, entre outros, podendo tornar a quimioterapia mais aversiva do que o próprio câncer. ${ }^{6}$

Com efeito, espera-se que as pessoas vivendo com câncer e, em especial aquelas submetidas à quimioterapia, mobilizem recursos psicossociais para lidar de forma adaptativa com o nível de estresse decorrente da doença oncológica ${ }^{7}$, bem como com os desconfortos relacionados ao tratamento quimioterápico. A esse processo, dá-se o nome de coping, podendo ainda estar associado ao uso da religião, espiritualidade ou fé (coping religioso-espiritual).

Coping é um construto que significa "lidar com", "manejar" ou "adaptar-se" definem como um conjunto de esforços cognitivos e comportamentais, utilizados pelos indivíduos com o objetivo de lidar com demandas específicas, internas ou externas, que surgem em situaçóes com potencial para desencadear estresse. O estresse é um processo fisiológico, psicológico e sociocultural que ocorre quando situaçóes da vida são percebidas pelo indivíduo como ameaçadoras ou desafiantes, desencadeando respostas adaptativas e de enfrentamento $^{1}$, podendo atingir níveis significativos quando essas situaçóes são avaliadas como excessivas aos recursos pessoais disponíveis ${ }^{1,9}$.
As estratégias de coping englobam um leque de açóes comportamentos e pensamentos - que, de acordo com a sua função, podem ser classificadas em dois tipos: focado na emoçáo e focado no problema. No coping focalizado na emoção, há um esforço significativo para regulação da resposta emocional relacionada ao evento estressor que a desencadeou. No coping focalizado no problema, há uma resposta concreta à situaçáo que deu origem ao estresse, alterando aspectos da relação entre a pessoa e o ambiente que mobiliza a tensão?

Entre esses esforços para lidar com situaçôes estressantes, existem aqueles relacionados à espiritualidade/ religiosidade, denominados de coping religioso/espiritual (CRE). O CRE é definido como o uso da religiáo, da espiritualidade ou fé para lidar com o estresse, podendo facilitar a solução de problemas, prevenir ou aliviar emoçóes negativas decorrentes de eventos estressantes ${ }^{10}$. A literatura aponta que o CRE pode estar associado tanto a estratégias orientadas para o problema quanto para a emoção, bem como à liberação de sentimentos negativos relacionados ao estresse, podendo apresentar caráter não adaptativo, por isso o CRE pode ser positivo ou negativo. Positivo quando proporciona efeito benéfico ao praticante (ex.: buscar a proteção de Deus; ajuda/conforto na religião); negativo, quando gera consequências prejudiciais ao indivíduo (ex.: avaliar o estressor como punição divina, delegar a Deus a resolução dos problemas) ${ }^{10}$. Em uma cultura dominada pela religiosidade, especialmente de origem Cristã como a da América Latina, onde o Brasil se inclui, o CRE é amplamente utilizado como estratégia para minimizar o estresse causado por uma patologia, na busca pelo senso de controle, manutenção da esperança, e propósito de vida.

Panzini e Bandeira ${ }^{10}$, em um artigo de revisão de literatura sobre o CRE, sintetizaram diversos estudos encontrados sobre a relação entre CRE e saúde física ou mental em diferentes grupos e condiçóes clínicas. Encontraram que, em homens HIV-soropositivos, alguns tipos de CRE estiveram associados a menos sintomas de depressão e à maior contagem de CD4. Em idosos hospitalizados, o CRE esteve associado a menores sintomas cognitivos e de depressão, menos sentimentos de infelicidade, fracasso e desesperança.

Mais recentemente, Carvalho et al. ${ }^{11}$ avaliaram o efeito da prece sobre a ansiedade de pacientes com câncer em tratamento quimioterápico, demonstrando uma relaçáo inversa entre ansiedade e religiosidade não organizacional; ou seja, aquela referente a atividades religiosas individuais. Concluíram que quanto maior o envolvimento espiritual do paciente, menor a ansiedade. A prece nesse contexto foi eficaz na redução de ansiedade em pacientes oncológicos. 
Estudo feito por Ottati e Campos ${ }^{12}$, cujo objetivo consistiu em verificar a relação entre a percepção de qualidade de vida e as estratégias de enfrentamento em 42 pacientes em tratamento quimioterápico, revelou que os indivíduos que estavam em fase inicial do tratamento fizeram maior uso de estratégias que modificam ou alteram o evento estressor (o tratamento oncológico) com o objetivo de controlar ou lidar melhor com a situação (estratégias de enfrentamento focalizadas no problema), além de se envolverem mais com práticas religiosas para auxiliar o processo de enfrentamento (busca por práticas religiosas), como também apresentaram uma percepção maior sobre sua condição afetiva e/ou cognitiva (domínio psicológico).

Apesar de o CRE corresponder a um construto que vem paulatinamente sendo objeto de estudos na área da saúde $e^{8,10,13-15}$, ainda são perceptíveis lacunas no que diz respeito à sua correlação com o tratamento oncológico, sobretudo o quimioterápico e efeitos adversos. Nesse sentido, o presente estudo poderá contribuir para a investigação no campo das estratégias de CRE utilizadas por pacientes oncológicos, especialmente no que diz respeito ao lidar com as toxicidades decorrentes da quimioterapia. Além disso, poderá ser útil à promoção de autoconhecimento por parte dos doentes e, não obstante, ao planejamento de intervençôes terapêuticas que incluam a dimensão religiosa/espiritual.

O objetivo deste trabalho consistiu em investigar a relação entre o uso do CRE e as respostas orgânicas às toxicidades da quimioterapia. Os objetivos específicos foram: a) traçar o perfil clínico e sociodemográfico dos pacientes oncológicos em tratamento quimioterápico atendidos na Unidade de Alta Complexidade em Oncologia (Unacon) de um Hospital Universitário; b) identificar os tipos de coping mais prevalentes entre os participantes; c) descrever as respostas orgânicas mais frequentes relacionadas às toxicidades da quimioterapia; d) avaliar a relação existente entre o CRE e as respostas orgânicas às toxicidades da quimioterapia.

\section{MÉTODO}

Trata-se de um estudo analítico, do tipo observacional, com corte transversal e delineamento correlacional acerca das relaçóes entre o uso do CRE e a quantidade de respostas orgânicas às toxicidades da quimioterapia. $\mathrm{O}$ projeto foi aprovado pelo Comitê de Ética em Pesquisa sob o Protocolo $\mathrm{n}^{\circ} 2.095 .070$, em consonância com as diretrizes preconizadas pelas resoluçóes no 466/12 e 510/2016 do Conselho Nacional de Saúde que normatizam a realização de pesquisas envolvendo seres humanos.

A amostra foi composta por 40 pacientes com diagnóstico oncológico, de ambos os sexos, que realizavam quimioterapia antineoplásica intravenosa na Unacon, de junho a agosto de 2017, selecionados de forma não probabilística intencional, conforme os critérios de inclusão/exclusão.

Utilizaram-se como critério de inclusão idade igual ou superior a 18 anos e o diagnóstico confirmado de neoplasia maligna em tratamento quimioterápico regular na Unacon. Foram excluídos os que apresentaram alteraçōes na consciência e na orientação temporal/espacial, bem como qualquer outra impossibilidade na compreensão dos instrumentos no momento da aplicação. Na sequência, foram convidados a participar voluntariamente do estudo, concordando e assinando o Termo de Consentimento Livre e Esclarecido (TCLE).

Os dados foram coletados por meio de dois instrumentos. Um questionário construído pelos pesquisadores com o intuito de obter dados clínicos e sociodemográficos e a escala de coping religioso/espiritual abreviada (CRE-Breve) traduzida, adaptada e validada para o Brasil por Panzini ${ }^{16,17}$. A CRE-Breve visa a mensurar o tipo e nível de CRE utilizado perante a experiência do tratamento quimioterápico. A escala está composta por 49 itens; dos quais, 34 dizem respeito ao CREP positivo e 15 ao negativo.

A abordagem aos pacientes ocorreu nas dependências da sala de quimioterapia da Unacon. Procedeu-se com orientaçóes aos potenciais participantes quanto ao objetivo do estudo, seus direitos (incluindo o da recusa) e à não interferência no tratamento. Optou-se ainda por entrevistas face a face, com facilitação do pesquisador na aplicação dos itens dos questionários, sem interferência na interpretação das respostas. Contudo, alguns dados clínicos como, por exemplo, o estadiamento da doença, foram obtidos no prontuário.

Os dados foram analisados de forma quantitativa, utilizando-se estatística descritiva para a caracterizaçáo dos participantes quanto a dados sociodemográficos e de saúde e o coeficiente de correlação de Spearman para testar as correlaçóes entre o nível de coping religioso e a frequência de toxicidades. O nível de CRE foi avaliado conforme a análise proposta pelas autoras a partir dos índices de CRE positivo e negativo.

\section{RESULTADOS}

Os resultados foram organizados em quatro sessóes, de acordo com os objetivos específicos: perfil sociodemográfico e clínico, prevalência de CRE, prevalência de toxicidades e relação entre nível de CRE e frequência de toxicidades.

\section{Perfil sociodemográfico e CLínico}

Entre os participantes, 55\% eram homens; as idades variaram entre 20 e 75 anos $(M=49,9 ; \mathrm{DP}=16,4)$; 
metade residia no município de Belém; $60 \%$ possuíam companheiro(a), sendo casados ou vivendo em união estável; $50 \%$ tinham apenas o ensino fundamental e somente $7,5 \%$, o ensino superior; $70 \%$ possuíam filhos, sendo dois em média. A renda familiar predominante foi de um salário mínimo (62,5\%).

Quanto à variável religiosidade, $50 \%$ dos entrevistados se declararam católicos e $32,5 \%$ evangélicos; $50 \%$ revelaram adotar práticas religiosas/espirituais frequentemente e apenas $12,5 \%$, raramente. Em relação à magnitude da fé, $82,5 \%$ a consideraram forte e somente $2,5 \%$ fraca.

Entre os diversos diagnósticos encontrados, o mais frequente foi o câncer colorretal (30\%) seguido do câncer de estômago (20\%), conforme a Figura 1.

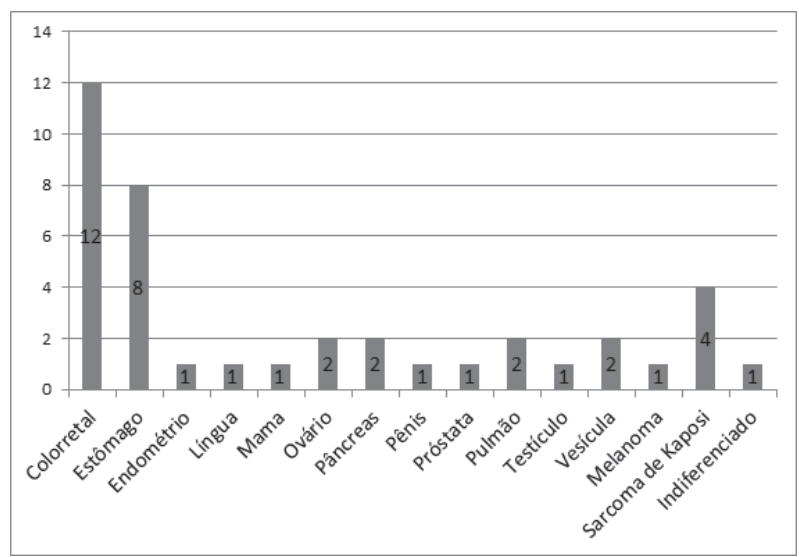

Figura 1. Prevalência dos tipos de câncer encontrados na Unacon/ HUJBB

O tempo de diagnóstico foi muito variável nesta amostra, indo de um a 48 meses $(M=12,7 ; \mathrm{DP}=11,97)$. No que se refere ao estágio da doença oncológica, $71 \%$ estavam no nível de estadiamento IV, ao passo que nenhum dos participantes se encontrava no estágio I. O número de sessôes de quimioterapia realizadas pelos pacientes também foi muito variável, indo de uma a 69 sessóes $(\mathrm{M}=16,9 ; \mathrm{DP}=16,71)$. A modalidade mais frequente de tratamento oncológico foi uma combinação de intervençáo cirúrgica com a quimioterapia antineoplásica, correspondendo a $52,5 \%$. Vale notar que $15 \%$ da amostra foi exposta a sessôes de radioterapia, além da cirurgia e da quimioterapia.

\section{PreVALÊNCIA DO COPING RELIGIOSO/ESPIRITUAL}

Para identificar os tipos de coping mais prevalentes, levou-se em consideração a existência das duas dimensões, coping religioso-espiritual positivo (CREP) e coping religioso-espiritual negativo (CREN), as quais foram avaliadas por meio de índices obtidos pela soma dos pontos na escala de respostas aos itens de cada dimensão.
No CREP, o índice variou de 34 a 170 (ponto médio=102); a média das respostas dos participantes foi de 112,25 (Mediana=113), indicando CREP um pouco acima do ponto médio do índice, o que sugere uso moderado do CREP. Já no CREN, o índice variou de 15 a 75 (ponto médio=45) com média de respostas igual a 30,3 (Mediana=30,5), um pouco abaixo do ponto médio do índice, o que também sugere uso moderado do CREN.

\section{PreValêNCIA DE TOXICIDADES}

Quanto às toxicidades da terapêutica quimioterápica, há uma diversidade de respostas orgânicas relacionadas, com frequências variadas. Para facilitar a análise, optou-se por agrupá-las em quatro categorias conforme a frequência de ocorrência entre os participantes: muito frequentes (apresentadas por $65 \%$ ou mais dos participantes), moderadamente frequentes (apresentadas por $40 \%$ a $64 \%$ dos participantes), pouco frequentes (apresentadas por $20 \%$ a $39 \%$ dos participantes) e raras (abaixo de $20 \%$ ), conforme ilustrado na Figura 2. Astenia foi a toxicidade mais frequente e sangramento, a menos frequente.

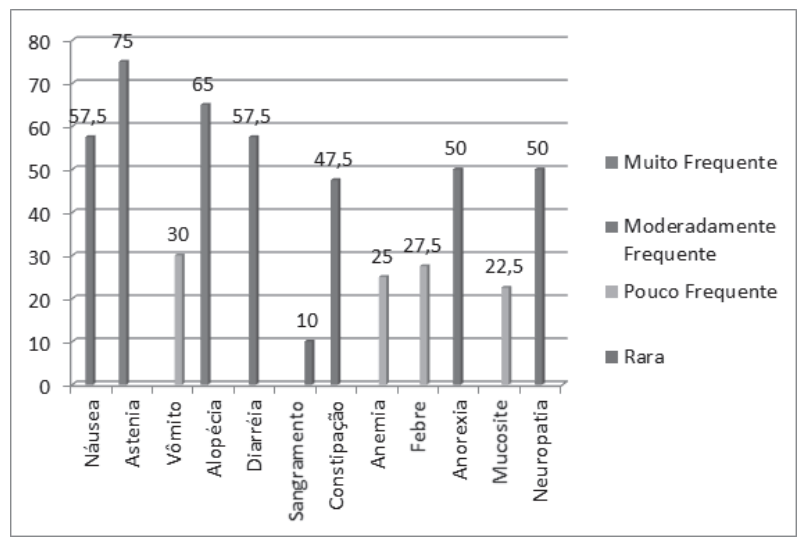

Figura 2. Frequência de toxicidades entre os pacientes submetidos à quimioterapia antineoplásica na Unacon/HUJBB, por categoria

\section{RELAÇÃO ENTRE COPING RELIGIOSO-ESPIRITUAL E TOXICIDADES}

O coeficiente de correlação de Spearman não foi estatisticamente significativo para a relaçáo entre a frequência de toxicidades e os índices de CREP e CREN. Contudo, quando se categorizou a frequência de toxicidades, o CREP e o CREN, e procedeu-se à análise por meio de tabelas cruzadas, surgiram algumas informaçōes interessantes.

Para efeito dessa análise, a frequência de toxicidades foi categorizada em inexistente (0), baixa (1 a 4), moderada (5 a 8) e elevada (9 a 12). O CREP foi categorizado em: a) uso baixo (34 a 68 pts.); moderado (69 a 135 pts.); e 
elevado (136 a 170 pts.). O CREN em uso baixo (15 a 30 pts.); moderado (31 a 59 pts.) e elevado (60 a 75 pts.).

Nas tabelas cruzadas (Tabelas 1 e 2), pode-se perceber a prevalência do uso moderado do CREP em todos os níveis de toxicidade. Cabe destacar que, para um paciente, o que corresponde a $25 \%$ da amostra, o grau elevado de toxicidade se mostrou associado ao uso elevado do CREP.

Quanto ao CREN, nenhum dos participantes fez uso elevado dessa estratégia. Em quase todos os graus de toxicidade, os participantes utilizaram o CREN em nível baixo, com exceção do grau moderado de toxicidade, em que $65 \%$ fizeram uso de CREN em nível moderado. Destaca-se que 75\% daqueles com grau elevado de toxicidade utilizaram o CREN em nível baixo.

\section{DISCUSSÃO}

Este estudo verificou a relação entre o uso do CRE e as respostas orgânicas às toxicidades da quimioterapia. Descreveram-se as características sociodemográficas e clínicas da amostra, apontou-se a prevalência de toxicidades e do uso do CREP e CREN e, finalmente, avaliou-se a relação entre o nível de CRE utilizado pelos participantes e a frequência de toxicidades apresentadas.

No que diz respeito à religiosidade, os resultados do presente estudo estão em consonância com os de Moreira-Almeida et al. ${ }^{18}$ que descreveram o envolvimento religioso na população brasileira e sua relação com variáveis sociodemográficas. Os autores revelaram que o nível de religiosidade entre os brasileiros é alto, variando de acordo com a região, a idade e o sexo. Mostraram, ainda, que $83 \%$ dos indivíduos consideraram a religiāo muito importante em suas vidas. No presente estudo, apenas $12 \%$ dos participantes exercitavam sua religiosidade/espiritualidade raramente e $82 \%$ consideraram a sua fé forte.

Com relação às características clínicas, os diagnósticos de câncer colorretal, de estômago e sarcoma de Kaposi foram bem expressivos nessa amostra. Em contraste, os cânceres de mama e próstata estão entre os menos frequentes. Porém, isso se deve ao fato de que a clientela da Unacon é, em parte, encaminhada da clínica de cirurgia, que realiza um número significativo de cirurgias oncológicas do aparelho digestivo, enquanto outra parte é proveniente das clínicas de pneumologia e infectologia, que são referências no hospital universitário. Por essa razão, os cânceres de mama e próstata, embora com considerável prevalência na Região Norte ${ }^{18}$ são referenciados para o Centro de Assistência de Alta Complexidade em Oncologia (Cacon) do Estado do Pará.

$O$ perfil sociodemográfico da amostra estudada, cuja renda familiar predominante foi de um salário mínimo $(62,5 \%)$ e o câncer de estômago foi o segundo mais frequente, está em conformidade com os dados do Instituto Nacional de Câncer José Alencar Gomes da Silva (INCA) ${ }^{19}$, que aponta uma relação entre condiçôes socioeconômicas insatisfatórias e a incidência dos cânceres do colo do útero e de estômago.

Tabela 1. Relação entre nível de toxicidade e grau de utilização do CRE positivo

\begin{tabular}{l|c|c|c|c|c}
\hline \multirow{2}{*}{ Nível de CREP } & \multicolumn{4}{c}{ Grau de toxicidade } & Total \\
\cline { 2 - 6 } & Inexistente & Baixo & Moderado & Elevado & 1 \\
\hline \multirow{2}{*}{ Uso baixo } & 0 & 1 & 0 & 0 & $2,5 \%$ \\
\hline \multirow{2}{*}{ Uso moderado } & $0,0 \%$ & $6,7 \%$ & $0,0 \%$ & $0,0 \%$ & 34 \\
\hline \multirow{2}{*}{ Uso elevado } & 1 & 12 & 18 & 3 & $85,0 \%$ \\
\hline \multirow{2}{*}{ Total } & $100,0 \%$ & $80,0 \%$ & $90,0 \%$ & $75,0 \%$ & 5 \\
\cline { 2 - 6 } & $0,0 \%$ & $13,3 \%$ & $10,0 \%$ & $25,0 \%$ & $12,5 \%$ \\
\hline
\end{tabular}

Tabela 2. Relação entre nível de toxicidade e grau de utilização do CRE negativo

\begin{tabular}{|c|c|c|c|c|c|}
\hline \multirow{2}{*}{ Nível de CREN } & \multicolumn{4}{|c|}{ Grau de toxicidade } & \multirow{2}{*}{ Total } \\
\hline & Inexistente & Baixo & Moderado & Elevado & \\
\hline \multirow{2}{*}{ Uso baixo } & 1 & 9 & 7 & 3 & 20 \\
\hline & $100,0 \%$ & $60,0 \%$ & $35,0 \%$ & $75,0 \%$ & $50,0 \%$ \\
\hline \multirow{2}{*}{ Uso moderado } & 0 & 6 & 13 & 1 & 20 \\
\hline & $0,0 \%$ & $40,0 \%$ & $65,0 \%$ & $25,0 \%$ & $50,0 \%$ \\
\hline \multirow{2}{*}{ Total } & 1 & 15 & 20 & 4 & 40 \\
\hline & $100,0 \%$ & $100,0 \%$ & $100,0 \%$ & $100,0 \%$ & $100,0 \%$ \\
\hline
\end{tabular}


A prevalência de toxicidades neste estudo foi considerável, o que de certo modo é esperado. Ferreira Filho $^{19}$ ressalta que as doses efetivas dos fármacos quimioterápicos frequentemente são proporcionais aos seus efeitos tóxicos. Contudo, essas toxicidades da quimioterapia dependem do agente antineoplásico utilizado e da capacidade de metabolizar e excretar os quimioterápicos, entre outros fatores ${ }^{20,21}$.

Com relação ao CRE, todos os participantes o utilizaram para lidar com o tratamento quimioterápico e suas toxicidades, fazendo uso tanto de estratégias positivas quanto negativas, assim como do coping focalizado no problema e nas emoçôes, corroborando os resultados de outros estudos similares ${ }^{14,22}$. Antoniazzi et al. ${ }^{23}$ argumentaram, com base em diversos estudos, que ambas as estratégias - focadas no problema e na emoção podem ser funcionais e complementares, sendo utilizadas na maioria dos episódios estressantes. Aquelas focadas na emoção podem facilitar as orientadas ao problema por reduzir a tensão; em contrapartida, as estratégias focadas no problema podem diminuir a ameaça, reduzindo assim o estresse emocional.

Veit e Castro ${ }^{15}$ mostraram que os índices de CRE estavam associados a múltiplas intervençóes terapêuticas e a longos períodos de tratamento; quanto mais o paciente oncológico era exposto a procedimentos invasivos, mais frequente era o uso do CRE, independentemente das estratégias empregadas, se positiva ou negativa. Os dados do presente estudo oferecem apoio aos de Veit e Castro $^{15}$, visto que $52 \%$ dos pacientes foram submetidos a uma combinação terapêutica de cirurgia oncológica e quimioterapia; o número de sessôes de quimioterapia realizadas foi de 16,9 em média e as duas estratégias de CRE foram utilizadas em nível moderado.

O uso moderado do CRE encontrado nessa amostra, por meio dos índices de CREP e CREN, foi considerado adequado e funcional, na medida em que estratégias de CREP estão associadas à otimização da sensação de bem-estar, evocação de sentimentos de esperança, confiança e segurança, podendo facilitar o acesso às redes de suporte $\mathrm{e}$ integraçáo social ${ }^{24}$. Por outro lado, as estratégias negativas podem causar danos ${ }^{24}$ quando utilizadas excessivamente, podendo impactar em níveis insatisfatórios de qualidade de vida e em índices expressivos de depressão ${ }^{25}$. Por exemplo, quando o paciente entende a doença como punição divina, sente-se excessivamente culpado ou tem absoluta crença na associação entre prece e cura e esta não acontece ${ }^{24}$.

Os participantes deste estudo, que apresentaram níveis elevados de toxicidade, fizeram baixo uso do CREN e uso moderado do CREP, contrapondo os resultados de Mesquita et al. ${ }^{14}$, em que os indivíduos que apresentaram efeitos colaterais intensos durante a quimioterapia mostraram tendência a utilizar mais o CREN. Contudo, conforme Mesquita et al. ${ }^{14}$, na medida em que aumentavam os eventos estressores, também aumentavam o uso das estratégias, tanto positivas quanto negativas.

Com base nos resultados, é possível afirmar que: a) De um modo geral, o CRE, tanto positivo como negativo, foi moderadamente utilizado pelos participantes, independente do grau de toxicidade; b) O CREN foi utilizado apenas em níveis baixo e moderado, não havendo uso dessa estratégia em nível elevado nos diferentes graus de toxicidade; c) O nível baixo de CREN predominou nos diferentes graus de toxicidade, exceto no grau moderado, no qual predominou o nível moderado de CREN.

Conclui-se que os pacientes com câncer submetidos à quimioterapia fazem uso tanto de estratégias de CREP como de CREN, sendo o CREP utilizado em maior intensidade. O CRE é um recurso que os pacientes oncológicos em quimioterapia antineoplásica regular dispóem e utilizam em uma tentativa de lidar com os eventos estressores relacionados a essa modalidade de tratamento e seus efeitos, incluindo as toxicidades que normalmente causam desconforto físico e psíquico.

Os resultados deste estudo chamam atenção para a necessidade de legitimação e integração da dimensão espiritual nos processos de saúde e doença, de modo a favorecer as formas de lidar com o sofrimento e a adaptação ao tratamento, especialmente de pessoas convivendo com doenças ameaçadoras à vida, como o câncer, e tratamentos invasivos, como o quimioterápico, que é potencialmente estressor. Espera-se, assim, que o paciente seja concebido em uma perspectiva biopsicossocioespiritual, sem desconsiderar a interferência e as repercussōes das crenças religiosas na forma em que as situações adversas serão avaliadas e administradas.

Quanto às limitaçóes deste estudo, pode-se ponderar a carência de instrumentos para medir o CRE em língua portuguesa. Desse modo, optou-se por utilizar a escala de CRE-Breve ${ }^{16,17}$ que, por ser composta por 49 itens, tornou sua aplicação exaustiva, particularmente no momento das sessôes de quimioterapia, além de prolongar o tempo de aplicação do instrumento, o que contribuiu para o tamanho reduzido da amostra no período disponível para a coleta dos dados (três meses).

\section{CONCLUSÃO}

Espera-se que este estudo contribua para instrumentalizar os profissionais de saúde no reconhecimento da dimensão espiritual como parte constituinte da subjetividade dos indivíduos que, no contexto de adoecimento, adversidades ou mesmo em situaçóes rotineiras, recorrem ao CRE para conviver e administrar demandas específicas. 


\section{CONTRIBUIÇÕES}

Leomar Santos Moraes Filho trabalhou na concepção e elaboração do projeto, na coleta de dados, na interpretaçáo dos dados, na redaçáo do artigo e revisão final, além de ser responsável por todos os aspectos do trabalho na garantia da exatidão e integridade de qualquer parte da obra. Hilma Tereza Tôrres Khoury trabalhou na orientação teórica e metodológica do projeto, na análise e interpretação dos dados, na redação do artigo e na revisão final.

\section{DECLARAÇÃO DE CONFLITO DE INTERESSES}

Nada a Declarar.

\section{REFERÊNCIAS}

1. Straub RO. Psicologia da Saúde: uma abordagem psicossocial. 3. ed. Porto Alegre: Artmed; 2014.

2. Instituto Nacional de Câncer José Alencar Gomes da Silva. $\mathrm{ABC}$ do câncer: abordagens básicas para o controle do câncer. Rio de Janeiro: INCA; 2011.

3. Guerra MR, Gallo CVM, Mendonça GAS. Risco de câncer no Brasil: tendências e estudos epidemiológicos mais recentes. Rev Bras Cancerol. 2005;51(3):227-34.

4. Ruppenthal JE. Toxicologia. Santa Maria: Universidade Federal de Santa Maria; 2013.

5. Riul S, Aguillar OM. Quimioterapia antineoplásica: revisão da literatura. Rev Min Enf. 1999;3(1/2):60-7.

6. Souza GG. Representações sociais do câncer para o familiar do paciente oncológico em tratamento quimioterápico [dissertação]. Rio de Janeiro: Universidade do Estado do Rio de Janeiro; 2011.

7. Peçanha DLN. Câncer: recursos de enfrentamento na trajetória da doença. In: Carvalho VA, Macieira RC, Liberato RP, Veit MT, Kovács MJ, Gomes MJB, et al., organizadores. Temas em psico-oncologia. São Paulo: Summus; 2008. p. 209-17.

8. Panzini RG, Bandeira DR. O coping religioso espiritual e a prática clínica. In: Santos FS, organizador. Arte de cuidar: saúde, espiritualidade e educação. São Paulo: Comenius; 2010. p. 62-74.

9. Lazarus RS, Folkman S. Coping and adaptation. In: Gentry WD, editores. The handbook of behavioral medicine. New York: Guilford; 1984. p. 282-325.

10. Panzini RG, Bandeira DR. Coping (enfrentamento) religioso/espiritual. Rev Psiquiatr Clín. 2007;34(Supl. 1):126-35.

11. Carvalho CC, Chaves ECL, Iunes DH, Simão TP, Grasselli CSM, Braga CG. A efetividade da prece na redução da ansiedade em pacientes com câncer. Rev Esc Enferm USP. 2014;48(4):683-9.

12. Ottati F, Campos MPS. Qualidade de vida e estratégias de enfrentamento de pacientes em tratamento oncológico. Act Colom Psicol. 2011;17(2):103-11.
13. Gobatto CA, Araújo TCCF. Coping religioso-espiritual: reflexôes e perspectivas para a atuação do psicólogo em oncologia. Rev. SBPH. 2010;13(1):52.

14. Mesquita AC, Chaves ECL, Avelino CCV, Nogueira DA, Panzini RG, Carvalho EC. A utilização do enfrentamento religioso/espiritual por pacientes com câncer em tratamento quimioterápico. Rev Latino-Am Enfermagem. 2013;21(2):1-7.

15. Veit CM, Castro EK. Coping religioso/espiritual e câncer de mama: Uma revisão sistemática da literatura. Psic Saúde \& Doenças. 2013;14(1):1-22.

16. Panzinni RG. Escala de coping religioso-espiritual (escala CRE): tradução, adaptação e validação da escala RCOPE, abordando relaçóes com saúde e qualidade de vida [dissertação]. Porto Alegre: Universidade Federal do Rio Grande do Sul; 2004.

17. Panzini RG, Bandeira, DR. Escala de coping religiosoespiritual (escala CRE 1): elaboração e validação de construto. Psicologia em Estudo. 2005;10(3):507-16.

18. Moreira-Almeida A, Pinsky I, Zaleski M, Laranjeira R. Envolvimento religioso e fatores sociodemográficos: resultados de um levantamento nacional no Brasil. Rev Psiq Clín. 2010;37(1):12-5.

19. Instituto Nacional de Câncer José Alencar Gomes da Silva. Estimativa 2016: incidência de câncer no Brasil. Rio de Janeiro: INCA; 2015.

20. Ferreira Filho AF. Conceitos gerais do câncer e do tratamento quimioterápico. In: Azevedo D, organizador. Psicooncologia e interdisciplinaridade: uma experiência na educação à distância. Porto Alegre: EdPUCRS; 2004. p. 59-71.

21. Caponero R, Lage LM. Temas em Psicooncologia. São Paulo: Summus; 2008.

22. Macieira RC. Avaliação da espiritualidade no enfrentamento do câncer de mama em mulheres [dissertação]. São Paulo: Universidade de Santo Amaro; 2007.

23. Antoniazzi AS. O conceito de coping uma revisão teórica. Estud Psicol. 1998;3(2):273-94.

24. Liberato RP, Macieira RC. Espiritualidade no enfrentamento do câncer. In: Carvalho VA, Macieira RC, Liberato RP, Veit MT, Kovács MJ, Gomes MJB, et al., organizadores. Temas em psicooncologia. São Paulo: Summus Editorial; 2008. p. 414-31.

25. Tarakeshwar N, Vanderwerker LC, Paulk E, Pearce MJ, Kasl SV, Prigerson HG. Religious coping is associated with the quality of life of patients with advanced cancer. J Palliat Med. 2006;9(3):646-57. 